\title{
Nonlinear Bloch-wave interaction and Bragg scattering in optically-induced lattices
}

\author{
Andrey A. Sukhorukov ${ }^{1}$, Dragomir Neshev ${ }^{1}$, Wieslaw Krolikowski ${ }^{2}$, and Yuri S. Kivshar ${ }^{1}$ \\ ${ }^{1}$ Nonlinear Physics Group and ${ }^{2}$ Laser Physics Center, \\ Center for Ultra-high bandwidth Devices for Optical Systems (CUDOS), \\ Research School of Physical Sciences and Engineering, \\ Australian National University, Canberra ACT 0200, Australif
}

\begin{abstract}
We study, both theoretically and experimentally, the Bragg scattering of light in optically-induced photonic lattices and reveal the key physical mechanisms which govern nonlinear self-action of narrow beams under the combined effects of Bragg scattering and wave diffraction, allowing for selecting bands with different effective dispersion.
\end{abstract}

PACS numbers: 42.25.Fx, 42.65.Sf, 42.79.Dj, 03.75.Kk

Dispersion and diffraction are the fundamental phenomena of wave physics, and they are responsible for temporal pulse spreading and beam broadening. The rate of wave broadening can be controlled in periodic structures where the effective geometric dispersion provides a key physical mechanism for manipulating waves in various physical systems, including the Bragg gratings in optical fibers [1], waveguide arrays 2], and Bose-Einstein condensates (BECs) in optical lattices [3] .

A strong change of the wave dispersion and diffraction occurs in the vicinity of spectrum band gaps where waves experience resonant Bragg scattering from a periodic structure 4]. In the structure shown in Fig. [1 both scattering and spreading depend on the angle between the beam and grating. For broad beams, the evolution of transmitted and scattered waves is defined by the effective dispersion parameters at the spectral peaks [5] , and can be studied in the effective-mass approximation [6]. The dynamics of narrow beams and wavepackets becomes more complicated, and it is strongly affected by nonlinearity, as recently discussed in Ref. 3. However, the key physical mechanisms which govern nonlinear self-action of narrow beams under combined effects of the Bragg scattering and wave diffraction remain largely unexplored.

In this Letter we study, both theoretically and experimentally, light propagation in optically-induced photonic lattices near the Bragg scattering angle. For the first time to our knowledge, we reveal generic relations between the Bloch-wave spectrum of the periodically modulated media and the specific structure of the diffracted waves describing analytically the key patterns of the beam scattering and self-action observed in our experiments. We also study the nonlinear Bloch-wave interaction and observe self-focusing in the spectrum gaps tracing the signatures of the Floquet-Bloch solitons.

Model. Essential features of light scattering in periodic photonic structures and the BEC dynamics in optical lattices can be described by similar mathematical models. For definiteness, we consider the optical beam propagation in a one-dimensional periodic structure described by

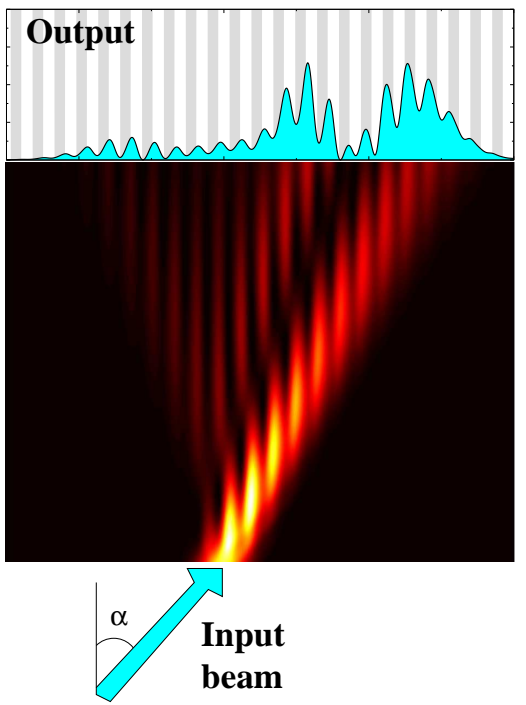

FIG. 1: Schematic of a laser beam propagating in a periodic photonic structure. The input Gaussian beam is split into several Bloch modes due to the Bragg scattering; top plot shows the output profile superimposed on top of the grating. Beam evolution is defined by the inclination angle $\alpha$.

the dimensionless equation,

$$
i \frac{\partial E}{\partial z}+D \frac{\partial^{2} E}{\partial x^{2}}+\nu(x) E+\mathcal{F}\left(x,|E|^{2}\right) E=0 .
$$

where $E(x, z)$ is the normalized electric field envelope, $x$ and $z$ are the transverse and propagation coordinates, normalized to the characteristic values $x_{s}$ and $z_{s}$, respectively, $D=z_{0} \lambda /\left(4 \pi n_{0} x_{0}^{2}\right)$ is the beam diffraction coefficient, $n_{0}$ is the average medium refractive index, and $\lambda$ is the vacuum wavelength. Function $\nu(x)$ characterizes the periodic modulation of the optical refractive index, $\nu(x+d) \equiv \nu(x)$, where $d$ is the spatial lattice period. The function $\mathcal{F}\left(x,|E|^{2}\right)$ defines the self-induced changes of the refractive index.

The theoretical analysis presented below is equally applicable to the BEC dynamics including the recent experimental results for the BEC dispersion management in optical lattices [3]. In this case, $E(x, z)$ stands for 


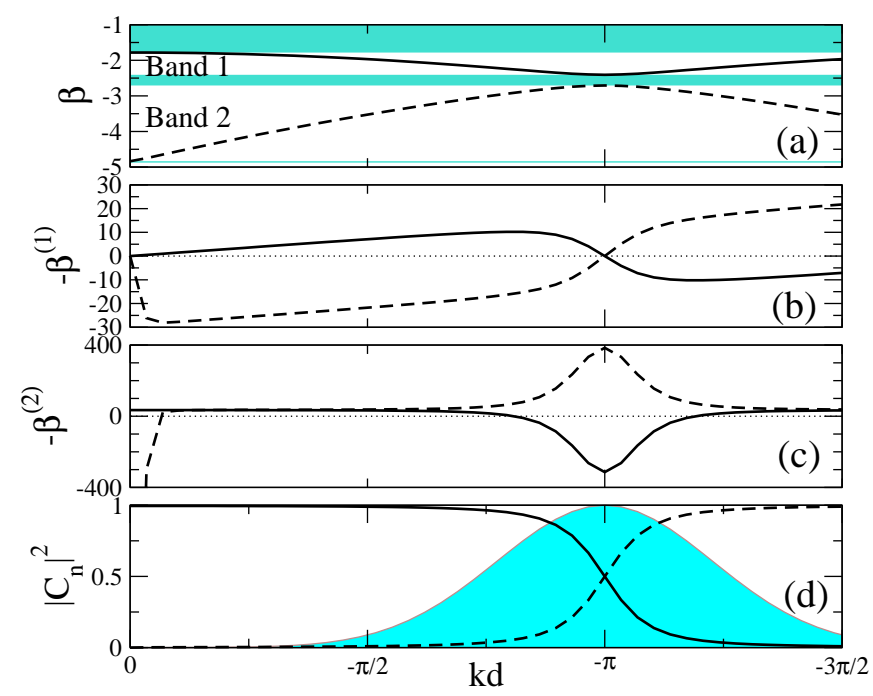

FIG. 2: (a) Dispersion curves for the Bloch waves in the first and second transmission bands. Shading marks the band gaps. (b,c) Propagation angle and diffraction coefficients of the Bloch waves. (d) Excitation coefficients of the Bloch waves; shading shows the normalized Fourier spectrum $\left(|F(k)|^{2}\right)$ of a beam incident at the Bragg angle. All parameters correspond to the experimental conditions.

the mean-field condensate wave function, $x$ is the spatial direction along a cigar-shaped trap, $z$ is the normalized time, and $D$ is a constant parameter. The periodic function $\nu(x)$ defines the effective potential of an optical lattice, and $\mathcal{F}\left(x,|E|^{2}\right) \simeq \gamma|E|^{2}$ characterizes the interatomic scattering.

General analytical approach. First, we present our general theory for describing scattering and diffraction of optical beams in periodic photonic structures [see Fig. 1] based on the Floquet-Bloch formalism [4]. The Bloch waves are special solutions of the linearized equation (1) of the form, $E_{\kappa, n}(x, z)=\psi_{\kappa, n}(x) \exp \left(i \kappa x+i \beta_{\kappa, n} z\right)$, where $\beta_{\kappa, n}$ and $\kappa$ are the Bloch-wave propagation constant and wavenumber, respectively, and the index $n=$ $1,2, \ldots$ marks the transmission band. The Bloch wave $\psi_{\kappa, n}(x)$ has the periodicity of the photonic structure, $\psi_{\kappa, n}(x+d) \equiv \psi_{\kappa, n}(x)$, and this constraint defines a specific dispersion relation $\beta_{\kappa, n}$, as sketched in Fig. 2(a). In a one-dimensional structure, the transmission bands are separated by gaps [4], where the wave propagation is forbidden ( $\kappa$ is real). The band-gaps are associated with Bragg reflection of light at certain incident angles.

The Bloch waves form a complete orthogonal set of eigenfunctions, and the linear evolution of an input beam in a periodic lattice is fully defined by its Bloch-wave spectrum $B(\kappa, n)$. Indeed, the electric field envelope $E$ can be found at a fixed $z$ as follows

$$
E(x, z)=\sum_{n} \int_{-\pi / d}^{+\pi / d} B(\kappa, n) \psi_{\kappa, n}(x) e^{i \kappa x+i \beta_{\kappa, n} z} d \kappa .
$$

The Bloch-wave spectrum can be linked to the Fourier spectrum of the input beam,

$$
B(\kappa, n)=\sum_{m} C_{n}(\kappa+2 \pi m / d) F(\kappa+2 \pi m / d),
$$

where $F(k)=(2 \pi)^{-1} \int_{-\infty}^{+\infty} E(x, 0) \exp (-i k x) d x$, and $C_{n}(\kappa)$ are the Bloch-wave excitation coefficients, as shown in Fig. 22 d).

In order to capture the essential features of the beam diffraction in a periodic lattice, we calculate the beam profiles in the far field regime. Assuming that $z$ exceeds several diffraction lengths, we use the stationary-phase approximation [7] to separate the leading-order contributions in the integral representation (2) and obtain

$$
E(x, z) \simeq \sqrt{\pi} \sum_{\kappa, n: x=-z \beta_{\kappa, n}^{(1)}} \frac{B(\kappa, n) \psi_{\kappa, n}(x)}{\left(\left|\beta_{\kappa, n}^{(2)}\right| z\right)^{1 / 2}} e^{i \varphi},
$$

where $\varphi=\kappa x+\beta_{\kappa, n} z+(\pi / 4) \operatorname{sign}\left(\beta_{\kappa, n}^{(2)}\right)$, and $\beta_{\kappa, n}^{(j)}=$ $\partial^{j} \beta_{\kappa, n} / \partial \kappa^{j}$. Solution (4) allows us to link the spatial profile of the diffracted beam and its Bloch-wave spectrum, and it provides a nontrivial generalization of the wellknown fact that in a homogeneous medium the profile of a diffracted beam maps directly its Fourier spectrum [7]. According to the summation condition in Eq. (4), $\beta_{\kappa, n}^{(1)}$ defines the propagation angle (or the group velocity) of the Bloch waves. The second derivative of the Blochwave dispersion, $\beta_{\kappa, n}^{(2)}$, characterizes the rate of the beam spatial divergence, and it has a meaning of the effective diffraction coefficient. In the regions where $\beta_{\kappa, n}^{(2)}<0$, the beam diffraction is normal (as in a homogeneous medium), and it is anomalous for $\beta_{\kappa, n}^{(2)}>0$. As illustrated in Figs. 2(a-c), the group velocity vanishes at the band edges, and it reaches a maximum absolute value inside the band, whereas the beam diffraction changes from normal to anomalous. A fundamental conclusion of our analysis is the following: at any given spatial location $x$, the beam profile is found as a superposition of Bloch waves from different spectral bands, and each of the bands gives two contributions associated with both normal and anomalous diffraction. Nonlinearity produces the opposite effects on such waves, resulting in either self-focusing or self-defocusing, and it is the competition of these effects that will define the beam shaping in periodic structures.

Equation (4) becomes formally invalid in the vicinity of a zero-diffraction point, which corresponds to a diffraction catastrophe. The corresponding contribution to the total field can be expressed through the Airy functions by taking into account higher-order diffraction terms. When $\beta_{\kappa, n}^{(2)}=0$, the general expression can be simplified, $-2 \pi z^{-1 / 3} B(\kappa, n) \psi_{\kappa, n}(x)\left|6 / \beta_{\kappa, n}^{(3)}\right|^{1 / 3}[\Gamma(-1 / 3)]^{-1}$. The contribution from the zero-diffraction region may become dominant in the far field where $B\left(\kappa_{1}, n\right)\left|z \beta_{\kappa_{1}, n}^{(3)}\right|^{1 / 3} \ll$ 
$B\left(\kappa_{2}, n\right)\left|z \beta_{\kappa_{2}, n}^{(2)}\right|^{1 / 2}$. This effect is responsible for the formation of a specific diffraction pattern with two side lobes in waveguide arrays 8]. In the case of dynamically induced gratings 9, 10], the refractive index contrast is smaller, and the spectral regions with near-zero diffraction are relatively narrow [see Fig. 2(c)] and are not expected to make a dominant contribution for experimentally feasible propagation lengths.

Experiment vs. theory. In order to study the Bragg scattering and Bloch-wave interaction experimentally, we create an optically-induced periodic lattice by interfering two ordinary-polarized coherent laser beams in a biased photorefractive crystal SBN:60, as discussed in Refs. 9, 10]. We apply an external electric field (up to $4000 \mathrm{~V} / \mathrm{cm}$ ) to the crystal, creating a periodic modulation of the optical refractive index through the electrooptic effect. In the SBN crystal, orthogonally polarized waves have significantly different electro-optic coefficients. We use the ordinary polarized interfering beams which propagate linearly and create a stable periodic lattice, whereas the extraordinary polarized probe beam experiences strong nonlinear self-action, which can be quantified through the nonlinear change of the refractive in$\operatorname{dex}, \nu(x)+\mathcal{F}\left(x,|E|^{2}\right)=-\gamma\left(I_{b}+I_{p}(x)+|E|^{2}\right)^{-1}$, where $I_{b}$ is the constant dark irradiance, $I_{p}(x)=I_{g} \cos ^{2}(\pi x / d)$ is the two-beam interference pattern which induces the periodic lattice, and $\gamma$ is the nonlinear coefficient which is proportional to the applied DC field. In order to match the conditions of our experimental observations in the theory and numerical calculations, we use the following parameters: $\lambda=0.532 \mu \mathrm{m}, n_{0}=2.4, x_{0}=1 \mu \mathrm{m}$, $z_{0}=1 \mathrm{~mm}, d=15.1, I_{b}=1, I_{g}=0.4, \gamma=2.13$, the crystal length is $L=15 \mathrm{~mm}$, and the input beam width is $21.16 \mu \mathrm{m}$. A detailed description of our experimental setup can be found in our earlier paper [10].

First, we perform numerical analysis of beam scattering in the linear regime using the exact Bloch-wave decomposition [Eq. (4)]. When the inclination angle of the scattered beam approaches that of the Bragg resonance, we observe the simultaneous excitation of several spectral bands by an input Gaussian beam, as illustrated in Figs. 31 a,b). These results are in perfect agreement with our general conclusion that a spatial profile of the diffracted beam maps various Bloch-wave spectral components. Indeed, three spectral regions can be excited simultaneously according to Fig. 2(d), where we superimpose the Fourier spectrum of the input beam on top of the Bloch-wave excitation coefficients.

In experiment, first we study the linear scattering when the beam power is low. Experimental results are summarized in Fig. 4 , where we show the dependence of the output beam profile on the tilt angle of the periodic lattice. Close to normal incidence, the first band is dominant, whereas at larger angles the excitation of the second band grows. In the vicinity of the Bragg angle [marked as a dashed line in Fig. 4(left)], the transmitted beam consists
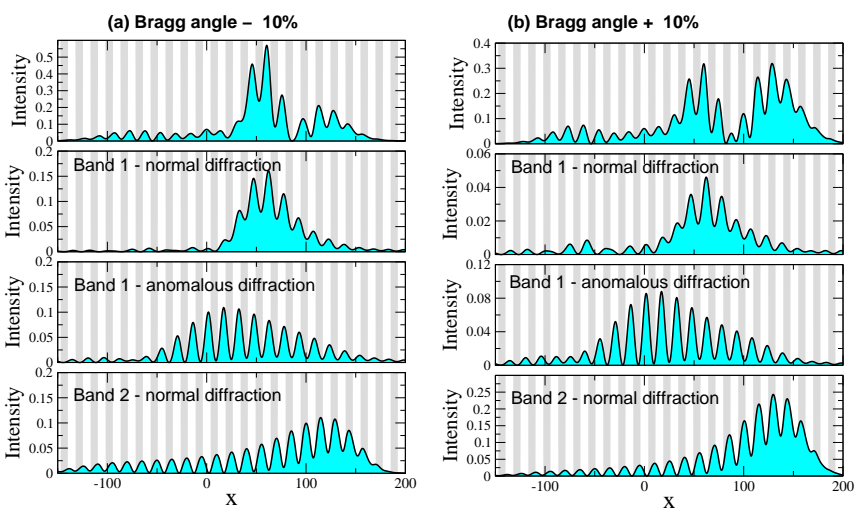

FIG. 3: Numerically calculated output beam profiles in the linear regime for an input Gaussian beam incident at the angles (a) $10 \%$ below and (b) $10 \%$ above the Bragg resonance. Intensity levels are normalized to the input peak intensity $I_{0}$. Shown is decomposition of the total intensity profile (top) between spectral regions with normal and anomalous diffraction of Bloch waves in the first and second bands (as indicated by labels). Shading marks the positions of the grating minima.

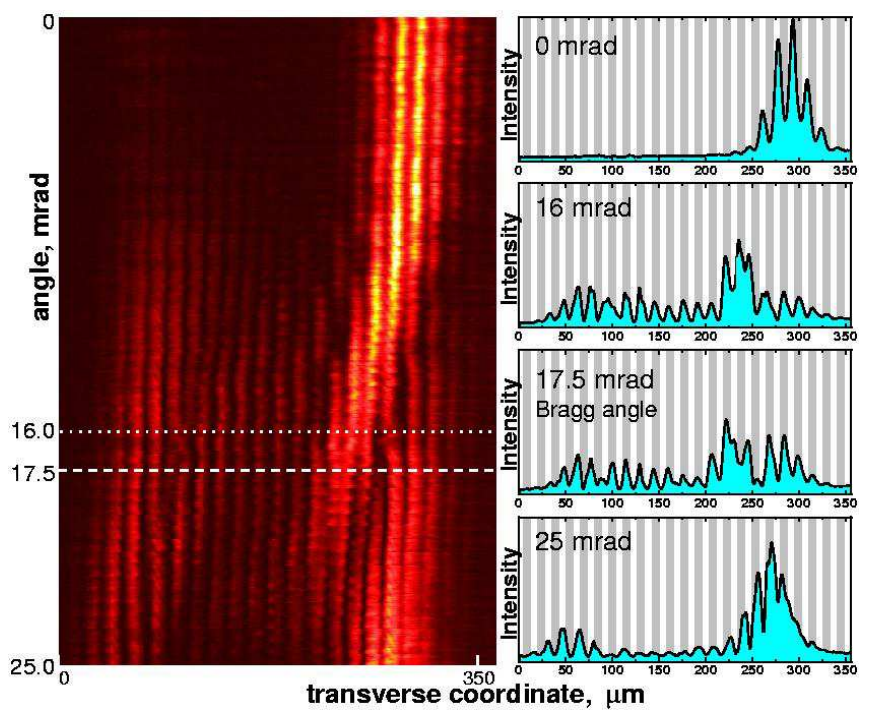

FIG. 4: Experimentally observed beam scattering by an optically-induced lattice for low laser powers. Left: output intensity vs. the tilt angle; right: beam profiles at different angles, shading marks the positions of the grating minima.

of two parts which correspond mainly to the excitation of the first and second bands, as predicted theoretically (cf. Fig. 3). For angles larger than the Bragg angle, the transmitted beam is composed mostly of the Bloch waves from the second spectral band. We note that the result of scattering very weakly depends on the initial beam position. This happens because the spectral width of the input beam is less than the width of the first Brillouin zone $(\Delta k<2 \pi / d)$, and according to Eq. (3) the beam shift introduces a uniform phase modulation of the Bloch-wave spectrum but does not change its shape. 


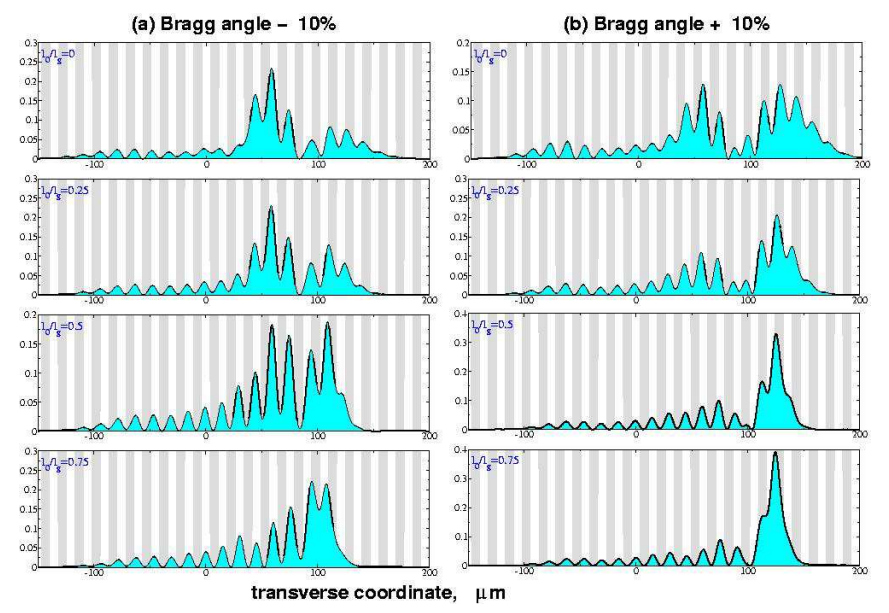

FIG. 5: Numerically calculated output beam profiles for different values of $I_{0}$. Beam parameters correspond to Fig. 3

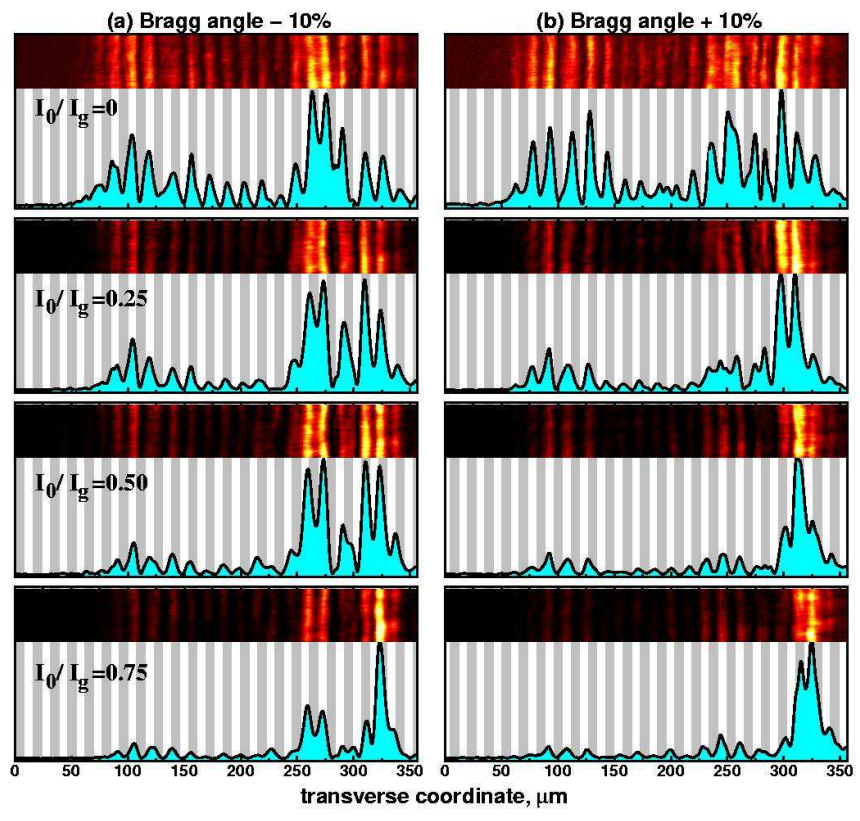

FIG. 6: Experimentally observed output beam profiles for different intensities, (a) below and (b) above the Bragg angle.

As the beam intensity grows, nonlinearity leads to strong changes in the output beam profiles, see results of numerical simulations of the full model Eq. (1) in Figs. [5 (a,b). In the nonlinear propagation regime, normally diffracting Bloch-wave components tend to selffocus whereas anomalously diffracting components experience self-defocusing. This fundamental property of the beam scattering suggests a possibility of performing nonlinear Bloch-wave spectroscopy of the optically-induced photonic lattices. In particular, we find that the beam self-action effect depends strongly on the incident an- gle. When the angle is below the Bragg resonance [see Figs. [5 (a)], the normally diffracting mode is dominant in the first band, see Fig. 31(a). Therefore, as the beam power grows, the mode experiences self-focusing. At the same time, we observe a strong nonlinearity-induced energy transfer into the right part of the beam, which corresponds to the excitation of the second band. In contrast, for the scattering angles above the Bragg resonance [see Figs. [5 (b)], the excitation of the second band is dominant, and in the first band anomalously-diffracting mode is stronger, see Fig. 3(b). This leads to defocusing on the left part of the transmitted beam, whereas the right part self-focuses. At higher powers, the beam starts erasing the optically-induced grating, and scattering is significantly reduced for both incident angles. In experiment, we increase the intensity of the probe beam, and observe the transformations of the beam profiles at the crystal output [see Fig. 6] in a remarkable agreement with the theoretical predictions [cf. Fig. 5]. Numerical calculations indicate that either one or two Floquet-Bloch solitons 11] can form under appropriate conditions, however the soliton formation distance exceeds the crystal length due to strong Bloch-wave interactions.

In conclusion, we have studied the Bragg scattering and nonlinear Bloch-wave inter-band interaction in optically-induced photonic lattices. We have established generic relations between the Bloch-wave spectrum and the structure of diffracted waves describing theoretically the specific patterns of the beam scattering and selfaction observed in experiment.

We thank B. Eggleton, E. A. Ostrovskaya, and C. M. de Sterke for useful discussions. This work was supported by the Australian Research Council.

* URL: http://www.rsphysse.anu.edu.au/nonlinear

[1] G. P. Agrawal, Nonlinear Fiber Optics (Academic Press, New York, 1988).

[2] H. S. Eisenberg et al., Phys. Rev. Lett. 85, 1863 (2000).

[3] B. Eiermann et al., Phys. Rev. Lett. 91, 060402 (2003).

[4] P. Yeh, Optical Waves in Layered Media (John Wiley \& Sons, New York, 1988).

[5] J. E. Sipe and H. G. Winful, Opt. Lett. 13, 132 (1988).

[6] N. W. Ashcroft and N. D. Mermin, Solid State Physics (Holt, Rinehart And Winston, New York, 1976).

[7] M. Born and E. Wolf, Principles of Optics: Electromagnetic Theory of Propagation, Interference and Diffraction of Light, seventh ed. (Cambridge University Press, UK, 2002).

[8] H. S. Eisenberg et al., Phys. Rev. Lett. 81, 3383 (1998).

[9] J. W. Fleischer et al., Phys. Rev. Lett. 90, 023902 (2003).

[10] D. Neshev et al., Opt. Lett. 28, 710 (2003).

[11] D. Mandelik et al., Phys. Rev. Lett. 90, 053902 (2003). 